\title{
A nomogram to predict risk factors of frequent defecation early after ileostomy reversal for rectal cancer patients
}

\author{
Jiaxin Deng ${ }^{1,2 \#}$, Mingli Su ${ }^{1,2 \#}$, Jiancong Hu ${ }^{1,2}$, Dezheng Lin ${ }^{1,2}$, Juan Li $^{1,2}$, Wei Liu ${ }^{1,2}$, Jiawei Zhang ${ }^{1,2}$, \\ Qinghua Zhong ${ }^{1,2}$, Xuefeng Guo ${ }^{1,2}$ \\ ${ }^{1}$ Department of Endoscopic Surgery, the Sixth Affiliated Hospital, Sun Yat-sen University, Guangzhou, China; ${ }^{2}$ Guangdong Provincial Key \\ Laboratory of Colorectal and Pelvic Floor Diseases, the Sixth Affiliated Hospital, Sun Yat-sen University, Guangzhou, China \\ Contributions: (I) Conception and design: X Guo, Q Zhong; (II) Administrative support: J Hu; (III) Provision of study materials or patients: D Lin, \\ J Li; (IV) Collection and assembly of data: W Liu, J Zhang; (V) Data analysis and interpretation: Jiaxin Deng, M Su; (VI) Manuscript writing: All \\ authors; (VII) Final approval of manuscript: All authors. \\ \#These authors contributed equally to this work. \\ Correspondence to: Xuefeng Guo. Department of Endoscopic Surgery, Sun Yat-sen University Sixth Affiliated Hospital, No. 26 Yuancun Erheng Road, \\ Guangzhou 510655, China. Email: guoxfeng@mail.sysu.edu.cn; Qinghua Zhong. Department of Endoscopic Surgery, Sun Yat-sen University Sixth \\ Affiliated Hospital, No. 26 Yuancun Erheng Road, Guangzhou 510655, China. Email: zhongqh3@mail.sysu.edu.cn.
}

\begin{abstract}
Background: Defecation disorders after ileostomy reversal are among the most common complications for rectal cancer patients, and significantly diminish their quality of life. The aim of this study was to identify the risk factors associated with frequent defecation early after ileostomy reversal for rectal cancer patients.

Methods: We retrospectively collected the data of rectal cancer patients who underwent ileostomy reversal from January 2018 to December 2019 at the Sixth Affiliated Hospital of Sun Yat-sen University. We divided patients into two groups: a no frequent defecation group ( $\leq 7$ times/day) and a frequent defecation group (>7 times/day).

Results: Of the 264 included patients, 59 patients (22.35\%) had frequent defecation within 1 month after ileostomy closure and were defined as the frequent defecation group. There were no significant differences between the two groups in terms of gender, age, hypertension, and diabetes. Univariate and multivariate analyses showed that proctitis $(\mathrm{P}=0.04)$ and anastomotic stenosis $(\mathrm{P}=0.02)$ were independent risk factors for frequent defecation. Four factors, including proctitis, anastomotic stenosis, hypertension, and age were included to construct the nomogram. The consistency index of the nomogram was 0.949 and the area under the curve value was 0.623 .

Conclusions: For rectal cancer patients, proctitis and anastomotic stenosis were correlated with frequent defecation within 1 month after ileostomy closure. Surgeons should pay attention to the rectal examination before ileostomy reversal surgery.
\end{abstract}

Keywords: Rectal cancer; ileostomy reversal; defecation; proctitis

Submitted Aug 17, 2021. Accepted for publication Oct 09, 2021.

doi: 10.21037/atm-21-4660

View this article at: https://dx.doi.org/10.21037/atm-21-4660

\section{Introduction}

Colorectal cancer (CRC) is one of the leading contributors to cancer-related morbidity and mortality worldwide $(1,2)$. In recent years, with the progress of multidisciplinary methods and the improvement of surgical strategies, the prognosis of colorectal patients has been significantly improved $(3,4)$. Sphincter-preserving surgery and preventive stoma surgery have become common procedures for rectal cancer patients. Data has shown that more than $70 \%$ of patients require a preventive stoma during anus preservation surgery (5). Ileostomy has a protective effect on the colon and rectum postoperatively, such as reducing the probability 
of anastomotic leakage, promoting the recovery of intestinal function, and alleviating enterogenous infection $(6,7)$. However, after ileostomy reversal, the indwelling colon and rectum may cause intestinal flora disorder, enteritis, pelvic floor dysfunction, and other manifestations, which significantly affect postoperative defecation function. It has been reported that more than $80 \%$ of rectal cancer patients may suffer from different degrees of defecation dysfunction, including incontinence, as well as urgent, frequent, and aggregation of defecation, which is collectively referred to as low anterior resection syndrome (LARS) $(8,9)$.

However, except for LARS, defecation disorder early after ileostomy reversal also obviously impairs the quality life of rectal cancer patients. The most common complications are postoperative ileus and frequent defecation (10). Several studies have shown that stimulation with probiotics before ileostomy closure could reduce the rate of postoperative ileus (11-14). Previous study showed that a nomogram can be established to predict long-term complications after patients receiving anterior resection (15). It should be noted that few studies have focused on defecation dysfunction early after ileostomy reversal, and the risk factors of frequent defecation among these patients remain unknown. It has been reported that nutrient deprivation can lead to dysbiosis and impaired intestinal renewal, which may play an important part in postoperative recovery (16).

Therefore, our retrospective study aimed to further explore the risk factors for frequent defecation of rectal cancer patients within 1 month after ileostomy closure. A nomogram was constructed to predict the incidence of frequent defecation.

We present the following article in accordance with the TRIPOD reporting checklist (available at https://dx.doi. org/10.21037/atm-21-4660).

\section{Methods}

\section{Patients}

Our study retrospectively collected the clinical data of patients with histologically confirmed CRC who underwent tumor resection with diverting stoma and received ileostomy reversal between January 2018 and December 2019 at the Sixth Affiliated Hospital of Sun Yat-sen University. The exclusion criteria were as follows: (I) patients with postoperative anastomotic leakage; (II) diagnosis with multiple malignant tumors at the time of enrollment; (III) patients with multiple postoperative stomas; and (IV) patients who had incomplete or missing follow-up data. The patients were divided into two groups according to the frequency of postoperative defecation: a frequent defecation group ( $>7$ times/day) and a no frequent defecation group ( $\leq 7$ times/day). The classification and pathological tumor node metastasis (TNM) staging were determined according to the American Joint Committee on Cancer (AJCC) guidelines. The timing of ileostomy reversal was determined by the clinical symptoms as well as findings from computed tomography (CT), magnetic resonance imaging (MRI), colonoscopy, and maximal squeeze pressure of the anus.

The main data retrieved by this study included age, gender, body mass index (BMI), concomitant disease, pathological tumor-node-metastasis (pTNM) stages, chemotherapy, radiotherapy, distance from the tumor to the anus, duration of stoma, and daily frequency of defecation after ileostomy reversal.

The study was approved by institutional ethics board of the Sixth Affiliated Hospital, Sun Yat-sen University (No. 2020ZSLYEC-139). We confirm that we have obtained ethical approval to conduct the study as well as permission from the dataset, and that the study was conducted in accordance with the provisions of the Declaration of Helsinki (as revised in Fortaleza, Brazil, October 2013). Because of the retrospective nature of the research, the requirement for informed consent was waived. The obtained data was only collected and analyzed; however, detailed patient information was not released publicly, and patient information confidentiality regulations were strictly adhered to.

\section{Definition of frequent defecation}

The definition of defecation frequency was mainly based on Low Anterior Resection Syndrome Score (LARSS). Normal defecation frequency is classed as experiencing between three bowel movements per week to three per day. In the present study, seven bowel movements per day was considered a more appropriate estimation of desirable bowel function. Therefore, stool frequency was categorized into the following baseline subgroups: a no frequent defecation group (no-FD group, $\leq 7$ times/day) and a frequent defecation group (FD group, $>7$ times/day). Put simply, the FD group comprised patients who had more than seven bowel movements per day.

\section{Definition of proctitis}

Proctitis is an inflammatory disorder that arises in the 
excluded rectum, including diversion proctitis, radiation proctitis, and ulcerative proctitis. Proctitis was cautiously defined by endoscopy or MRI/CT with mucosal lesion or circumferential thickening of the rectal wall with mural stratification, as well as diffuse edema of the mesorectum and pelvic soft tissue (17). Descriptions of endoscopic findings were focused on five criteria including congested mucosa, telangiectasia, ulceration, stricture, and necrosis (18).

\section{Definition of anastomotic stenosis}

Until now, no consensus has been reached about the definition of anastomotic stenosis. Generally, anastomotic stenosis is defined as failure to pass a $12-\mathrm{mm}$ scope through the anastomosis or the reduction of at least $2 / 3$ of the diameter (19). In this study, we defined rectal anastomotic stenosis as significant resistance or failure to pass through the anastomosis or the new rectum when using an index finger or $12 \mathrm{~mm}$ colonoscope, accompanied by clinical symptoms such as laborious defecation and anal incontinence.

\section{Statistical analysis}

IBM SPSS (version 26.0 for Windows; SPSS, Chicago, IL, USA) was used for data analysis. Measurement data are expressed as the mean \pm Standard deviation (SD) or median (minimum, maximum). Mann-Whitney rank sum tests or T-tests were used to compare the measurement data between groups. Logistic regressions were used to analyze the univariate and multivariate factors. Variables with a $\mathrm{P}$ value $<0.2$ in the univariate analysis were included into the multivariable model using logistic regression.

The following statistical procedures and model construction were performed using R software (version 4.1.0). The nomogram and calibration curve were constructed using the "rms" package. Receiver operating characteristic (ROC) curve analysis was carried out using the "pROC" package, and the Delong test was utilized to compare the differences between ROC curves. The Hosmer-Lemeshow Goodness of Fit (GOF) test was used to examine the fitness of the logistics regression model with a grouping of 10 .

\section{Results}

\section{Patients' characteristics}

The characteristics of the included patients are shown in Table 1. In this study, a total of 264 patients who underwent rectal cancer surgery and ileostomy closure were included. Of these, 59 patients $(22.35 \%)$ had frequent defecation within 1 month after ileostomy closure and were defined as the FD group. There was no significant different between the two groups in terms of gender, age, hypertension, and diabetes.

Eight patients $(13.56 \%)$ in the FD group had proctitis, which was more than that in the no-FD group ( $\mathrm{P}=0.029)$. The rate of anastomotic stenosis in the no-FD group was significantly lower than that in the FD group $(8.29 \%$ vs. $20.34 \%, \mathrm{P}=0.016$ ).

\section{Univariate and multivariate logistic regression analysis}

We used univariate and multivariate analyses to evaluate the risk factors of frequent defecation 1 month after ileostomy reversal in Table 2. Variables with a $\mathrm{P}$ value $<0.2$ including anastomotic stenosis $(\mathrm{P}=0.012)$, proctitis $(\mathrm{P}=0.016)$, hypertension $(\mathrm{P}=0.135)$, age $(\mathrm{P}=0.138)$, and sex $(\mathrm{P}=0.176)$ were included into the multivariable model using logistic regression. Multivariate analysis indicated that proctitis [hazard ratio (HR): 2.94, 95\% CI: 1.030-8.280, $\mathrm{P}=0.04$ ] and anastomotic stenosis (HR: 2.65, 95\% CI: 1.120-6.150, $\mathrm{P}=0.02$ ) were independent risk factors for frequent defecation.

In order to further comprehensively predict postoperative frequent defecation, we constructed a prediction model based on significant and predefined predictors. Preliminary analysis showed that anastomotic stenosis, proctitis, hypertension, age, and sex were associated with postoperative frequent defecation. Since there were numerous factors included in this prediction model and sex belongs to the binary variable, we designed ROC curves to test the prediction efficiency of each component. The results showed that there were no differences in the predictive ability of the two logistics models (with or without sex) (Figure 1). Accordingly, the other four variables were included finally.

Hosmer-Lemeshow Goodness of Fit (GOF) test analysis was performed, and the result showed that the model had noteworthy predictive potential ( $\mathrm{P}=0.949)$. In our nomogram, we drew a line for each variable to the points axis, added the determined scores to obtain the total score, and determined the predicted value for each component by drawing a vertical line from the total points axis (Figure $2 A$ ). Notably, the calibration curve (Figure $2 B$ ) showed good calibration of the nomogram and the area under the curve (AUC) value of the model was 0.623 (Figure 2C). The calibration curves of the predicted and observed values indicated that our model had moderate predictive ability for the incidence of frequent 
Table 1 Patient characteristics

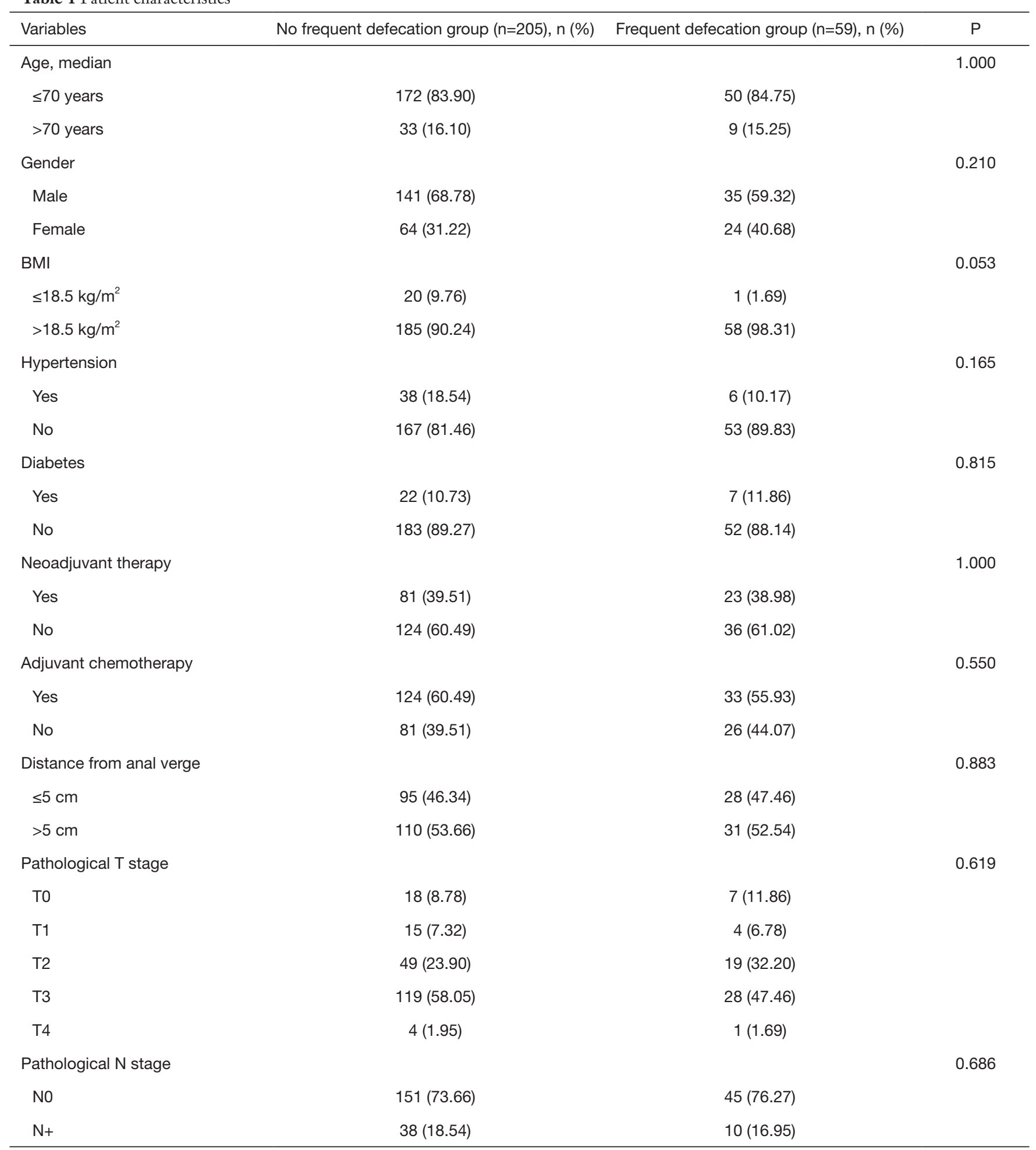

Table 1 (continued) 
Table 1 (continued)

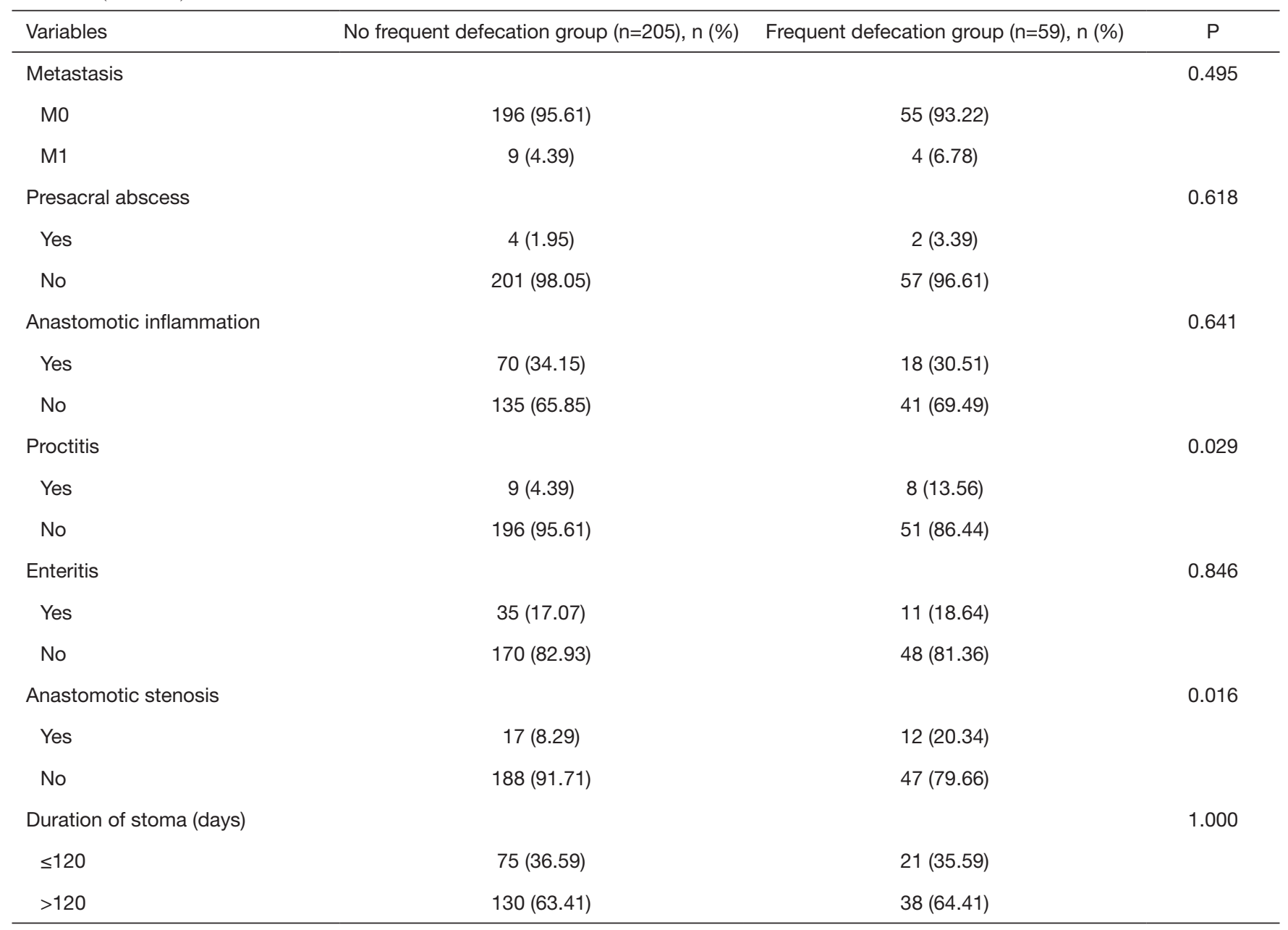

defecation.

In addition, radiotherapy may cause chronic damage to the rectum and affect the bowel function of rectal cancer patients. Thus, we performed a subgroup analysis according to neoadjuvant radiotherapy. As shown in Table 3, anastomotic stenosis was an independent risk factor of frequent defecation for patients who received neoadjuvant radiotherapy (HR: 0.063; 95\% CI: 0.006-0.689; $\mathrm{P}=0.024)$.

\section{Discussion}

In this study, we found that postoperative proctitis and anastomotic stenosis were independent risk factors of frequent defecation after ileostomy closure for CRC patients, which was consistent with the findings of previous studies. Generally, the two main kinds of inflammation of the colon and rectum for patients with ileostomy are colitis and proctitis. Diversion colitis is characterized by inflammation of the large bowel mucosa and up to $30 \%$ of patients remain asymptomatic (20). The main treatment for colitis is the restoration of the continuity of the digestive tract, including ileostomy reversal or stimulation of the efferent loop with probiotics prior to surgery (21). Several studies have shown that probiotics or nutrient solution stimulation 1-2 weeks prior to surgery could improve bowel function after ileostomy closure, which further confirm the reversibility of colitis (11-13,21). Similarly, in this study, we found that colitis was not a risk factor for frequent defecation 1 month after ileostomy closure. However, postoperative proctitis was relative to bowel diversion, rectal resection, radiotherapy, etc. and could affect defecation directly and persistently. 
Table 2 Univariate and multivariate logistic regression for the risk factors of frequent defecation

\begin{tabular}{|c|c|c|c|}
\hline Variables & Univariate $(\mathrm{P})$ & \multicolumn{2}{|c|}{ Multivariate } \\
\hline Age, median & 0.138 & $0.99(0.970-1.020)$ & 0.55 \\
\hline Gender & 0.176 & $1.40(0.750-2.600)$ & 0.29 \\
\hline BMI & 0.356 & - & - \\
\hline Diabetes & 0.806 & - & - \\
\hline Neoadjuvant chemotherapy & 0.942 & - & - \\
\hline Adjuvant chemotherapy & 0.530 & - & - \\
\hline Neoadjuvant radiotherapy & 0.244 & - & - \\
\hline Metastasis & 0.458 & - & - \\
\hline Presacral abscess & 0.519 & - & - \\
\hline Anastomotic inflammation & 0.602 & - & - \\
\hline Proctitis & 0.016 & $2.94(1.030-8.280)$ & 0.04 \\
\hline Enteritis & 0.779 & - & - \\
\hline Anastomotic stenosis & 0.012 & $2.65(1.120-6.150)$ & 0.02 \\
\hline Duration of stoma (days) & 0.889 & - & - \\
\hline
\end{tabular}

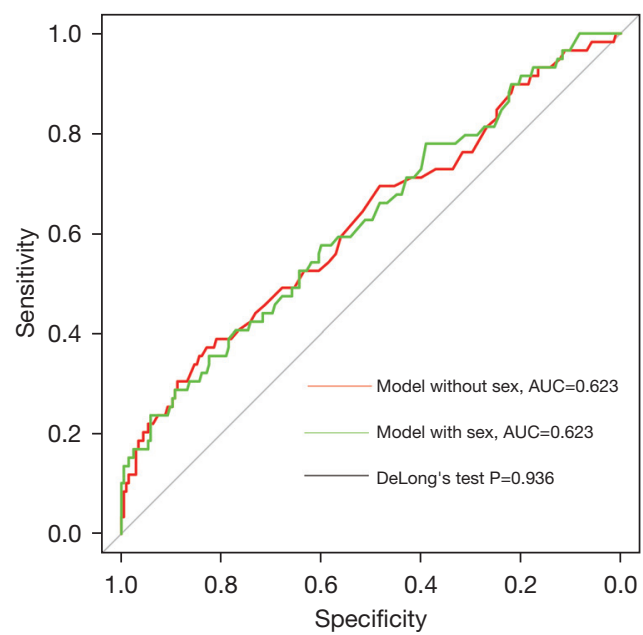

Figure 1 Receiver operating characteristic (ROC) curves of logistics models without sex (red curve) and with sex (green curve). The ROC curves were constructed by plotting sensitivity against specificity calculated by consecutive cut-offs in the range of predictive values of the logistics models. The Delong test showed that there was no difference in the predictive ability of the two logistics models.
Defecation dysfunction, such as low anterior resection syndrome (LARS), significantly affects the postoperative life quality of CRC patients. Previous studies have found that several risk factors, including preoperative neoadjuvant therapy, postoperative adjuvant chemotherapy, tumor location, and anastomotic leakage were associated with LARS (22-24). However, except for the long-term defecation situation, defecation disorder early after ileostomy closure also has a significant negative impact on CRC patients but is less concerned at present. Some measures were reported to improve the early defecation function after ileostomy closure, including pelvic floor muscle training (25), transanal probiotics enema (26), preperfusion of probiotics in the colon and rectum before ileostomy closure (27), and so on. Our study confirmed that proctitis is an independent risk factor for short-term postoperative defecation function, which can be intervened by paying more attention to the regulation of intestinal flora prior to ileostomy closure. However, more studies with larger sample size or prospective, randomized, controlled clinical trials are 

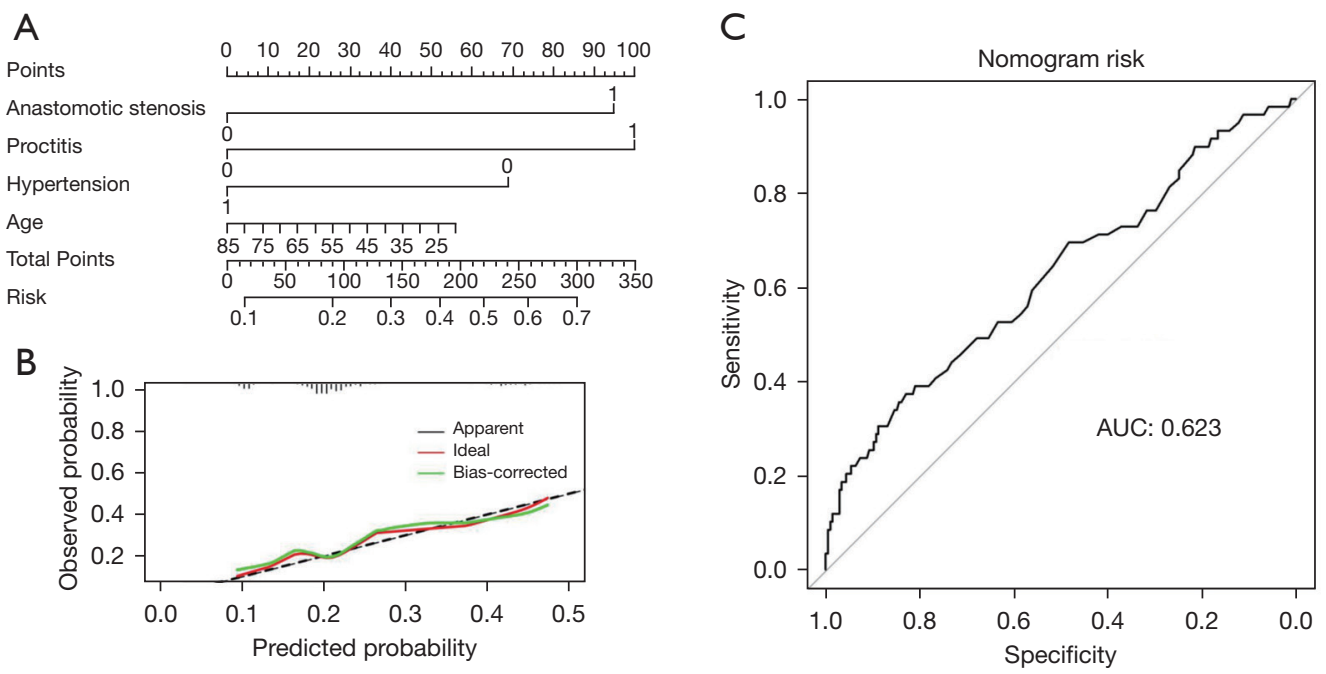

Figure 2 A four parameter nomogram was constructed (A) and the calibration curve (B) showed good calibration of the nomogram. The red and green lines represent the predicted and resampled (100 times) predicted probability of the nomogram model, respectively. The area under the curve of the nomogram is $0.623(\mathrm{C})$.

Table 3 Univariate and multivariate logistic regression for the risk factors of defecation dysfunction after neoadjuvant radiotherapy

\begin{tabular}{|c|c|c|c|}
\hline Variables & Univariate $(P)$ & \multicolumn{2}{|c|}{ Multivariate } \\
\hline Pathological N stage & 0.074 & - & 1.000 \\
\hline Anastomotic stenosis & 0.070 & $0.063(0.006-0.689)$ & 0.024 \\
\hline
\end{tabular}

needed to further confirm the effect and treatment of rectal complications such as proctitis and anastomotic stenosis.

Neoadjuvant chemoradiotherapy is an effective treatment for rectal cancer, which can remarkably improve the radical resection rate of the tumor, reduce the risk of local recurrence, and increase the opportunity of anal preservation. However, some complications may occur after radiotherapy, including rectal anastomotic leakage, anastomotic stenosis, and proctitis. Dulskas et al. included patients who had received rectal cancer resection at the National Cancer Institute of Lithuania from 2010 to 2015, and found that preoperative chemoradiotherapy was confirmed to be a risk factor for postoperative defecation changes, which was similar to the results of many previous studies (28). Sun et al. also showed that long-course neoadjuvant radiation, along with low anastomosis, are likely independent risk factors for postoperative bowel function and quality of life (QOL) (29). Anastomotic stenosis is a common complication after rectal resection, with an incidence in the range of 3-30\% (30). Numerous factors, such as neoadjuvant radiotherapy, diverting stoma, and anastomotic leakage, have been reported as risk factors for stenosis (31). In this study, patients with leakage were excluded, and in order to further study the effect of radiotherapy on postoperative defecation function, we separately screened patients who had received preoperative radiotherapy. Notably, anastomotic stenosis was an independent risk factor for defecation frequency, which was consistent with previous research findings $(30,32)$. In the early postoperative period after rectal resection, the common type of anastomotic stenosis is membranous stenosis, which can be treated by transanal dilation, such as digital rectal examination and dilation. Therefore, attention should be paid to the prevention and management of rectal complications early after rectal cancer surgery, and patients were ordered to return to the clinic for rectal examination within 1 month after surgery in our center.

A few studies have shown that age and tumor distance 
from the anal margin may be risk factors for defecation dysfunction. Sturiale et al. found that age and occurrence of postoperative LARS were statistically significant (33). Emmertsen et al. believed that the distance between the tumor and anal margin and the height of the anastomotic site notably affected the defecation function score of LARS (34). In our study, we found that age $[\mathrm{P}=0.024$, odds ratio (OR) $=2.605]$ and tumor location $(\mathrm{P}=0.018, \mathrm{OR}=1.861)$ affected the incidence of defecation frequency within 2 weeks after surgery. However, data analysis within 1 month after surgery showed that tumor location was no longer a risk factor for frequent defecation. It can be speculated that in the early stage after ileostomy closure, the lower the tumor location is, the easier it can be to stimulate the pelvic floor and induce increased frequency of defecation. After 1 month of recovery, the patient's pelvic floor muscle function will have partially recovered or the body will have become accustomed to it, so this effect is attenuated, thus making it no longer a risk factor. However, the pelvic floor muscle function will decline with age, so we also included age $(\mathrm{P}<0.02)$ as a risk factor into the nomogram prediction model.

In this study, a nomogram was developed to predict the accurate occurrence risk of frequent defecation early after ileostomy closure. To the best of our knowledge, few predictive models of defecation early after the restoration of the continuity of the digestive tract have been published. The concordance index (C-index) of our nomogram was 0.623 , which indicated moderate predictive ability. However, studies with large sample size are needed to improve the predictive potency.

Our study had several limitations that should be noted. Firstly, this was a retrospective study with a relatively small sample, and the data included were collected from the patients' medical records. However, the clinical characteristics were comparable between the two groups. Secondly, our study lacked a certain quantitative standard for proctitis, which made it difficult to reflect the severity of proctitis and evaluate it more intuitively. Thirdly, gut microflora has a significant influence on the defecation of rectal cancer patients, and detection and in-depth analysis of the microflora of enrolled patients is recommended in future studies. Therefore, further well-designed, larger sample size study are needed to identify the certain correlations between rectal complications and defection function.

\section{Conclusions}

In conclusion, our study showed that proctitis and anastomotic stenosis were correlated with frequent defecation within 1 month after ileostomy closure for rectal cancer patients. Surgeons should pay attention to the rectal examination before ileostomy reversal surgery.

\section{Acknowledgments}

Funding: This work was supported by the Science and Technology Planning Project of Guangzhou, China (201803010074).

\section{Footnote}

Reporting Checklist: The authors have completed the TRIPOD reporting checklist. Available at https://dx.doi. org/10.21037/atm-21-4660

Data Sharing Statement: Available at https://dx.doi. org/10.21037/atm-21-4660

Conflicts of Interest: All authors have completed the ICMJE uniform disclosure form (available at https://dx.doi. org/10.21037/atm-21-4660). The authors have no conflicts of interest to declare.

Ethical Statement: The authors are accountable for all aspects of the work in ensuring that questions related to the accuracy or integrity of any part of the work are appropriately investigated and resolved. The study was approved by institutional ethics board of the Sixth Affiliated Hospital, Sun Yat-sen University (No. 2020ZSLYEC-139). We confirm that we have obtained ethical approval to conduct the study as well as permission from the dataset, and that the study was conducted in accordance with the provisions of the Declaration of Helsinki (as revised in Fortaleza, Brazil, October 2013). Because of the retrospective nature of the research, the requirement for informed consent was waived. The obtained data was only collected and analyzed; however, detailed patient information was not released publically, and patient information confidentiality regulations were strictly adhered to.

Open Access Statement: This is an Open Access article distributed in accordance with the Creative Commons Attribution-NonCommercial-NoDerivs 4.0 International License (CC BY-NC-ND 4.0), which permits the noncommercial replication and distribution of the article with the strict proviso that no changes or edits are made and the 
original work is properly cited (including links to both the formal publication through the relevant DOI and the license). See: https://creativecommons.org/licenses/by-nc-nd/4.0/.

\section{References}

1. Sung H, Ferlay J, Siegel RL, et al. Global Cancer Statistics 2020: GLOBOCAN Estimates of Incidence and Mortality Worldwide for 36 Cancers in 185 Countries. CA Cancer J Clin 2021;71:209-49.

2. Qiu H, Cao S, Xu R. Cancer incidence, mortality, and burden in China: a time-trend analysis and comparison with the United States and United Kingdom based on the global epidemiological data released in 2020. Cancer Commun (Lond) 2021;41:1037-48.

3. Siegel RL, Miller KD, Goding Sauer A, et al. Colorectal cancer statistics, 2020. CA Cancer J Clin 2020;70:145-64.

4. Yang Z, Li Y, Qin X, et al. Development and Validation of a Prognostic Nomogram for Colorectal Cancer Patients With Synchronous Peritoneal Metastasis. Front Oncol 2021;11:615321.

5. Gao JL, An YB, Wang D, et al. Current status of research on short-term quality of life after sphincteric-saving surgery in rectal cancer patients. Zhonghua Wei Chang Wai Ke Za Zhi 2020;23:415-20.

6. Biagi JJ, Raphael MJ, Mackillop WJ, et al. Association between time to initiation of adjuvant chemotherapy and survival in colorectal cancer: a systematic review and metaanalysis. JAMA 2011;305:2335-42.

7. Sparreboom CL, van Groningen JT, Lingsma HF, et al. Different Risk Factors for Early and Late Colorectal Anastomotic Leakage in a Nationwide Audit. Dis Colon Rectum 2018;61:1258-66.

8. Battersby NJ, Bouliotis G, Emmertsen KJ, et al. Development and external validation of a nomogram and online tool to predict bowel dysfunction following restorative rectal cancer resection: the POLARS score. Gut 2018;67:688-96.

9. Keane C, Fearnhead NS, Bordeianou L, et al. International consensus definition of low anterior resection syndrome. Colorectal Dis 2020;22:331-41.

10. Garfinkle R, Savage P, Boutros M, et al. Incidence and predictors of postoperative ileus after loop ileostomy closure: a systematic review and meta-analysis. Surg Endosc 2019;33:2430-43.

11. Rodríguez-Padilla Á, Morales-Martín G, PérezQuintero R, et al. Postoperative Ileus after Stimulation with Probiotics before Ileostomy Closure. Nutrients
2021;13:626.

12. Abrisqueta J, Abellan I, Luján J, et al. Stimulation of the efferent limb before ileostomy closure: a randomized clinical trial. Dis Colon Rectum 2014;57:1391-6.

13. Garfinkle R, Trabulsi N, Morin N, et al. Study protocol evaluating the use of bowel stimulation before loop ileostomy closure to reduce postoperative ileus: a multicenter randomized controlled trial. Colorectal Dis 2017;19:1024-9.

14. Rodríguez-Padilla Á, Morales-Martín G, Pérez-Quintero $\mathrm{R}$, et al. Serological Biomarkers and Diversion Colitis: Changes after Stimulation with Probiotics. Biomolecules 2021;11:684.

15. Battersby, N.J., et al. Development and external validation of a nomogram and online tool to predict bowel dysfunction following restorative rectal cancer resection: the POLARS score. Gut 2018;67:688-96.

16. Beamish, E.L., et al., Loop ileostomy-mediated fecal stream diversion is associated with microbial dysbiosis. Gut Microbes 2017;8:467-78.

17. Cheng YK, Qin QY, Huang XY, et al. Effect of interval between preoperative radiotherapy and surgery on clinical outcome and radiation proctitis in rectal cancer from FOWARC trial. Cancer Med 2020;9:912-9.

18. Wachter S, Gerstner N, Goldner G, et al. Endoscopic scoring of late rectal mucosal damage after conformal radiotherapy for prostatic carcinoma. Radiother Oncol 2000;54:11-9.

19. Sartori A, De Luca M, Fiscon V, et al. Retrospective multicenter study of post-operative stenosis after stapled colorectal anastomosis. Updates Surg 2019;71:539-42.

20. Kabir SI, Kabir SA, Richards R, et al. Pathophysiology, clinical presentation and management of diversion colitis: a review of current literature. Int J Surg 2014;12:1088-92.

21. Rodríguez-Padilla Á, Morales-Martín G, Pérez-Quintero R, et al. Diversion Colitis: Macro and Microscopic Findings after Probiotics Stimulation. Biology (Basel) 2021;10:303.

22. Hughes DL, Cornish J, Morris C, et al. Functional outcome following rectal surgery-predisposing factors for low anterior resection syndrome. Int J Colorectal Dis 2017;32:691-7.

23. Hain E, Manceau G, Maggiori L, et al. Bowel dysfunction after anastomotic leakage in laparoscopic sphincter-saving operative intervention for rectal cancer: A case-matched study in 46 patients using the Low Anterior Resection Score. Surgery 2017;161:1028-39. 
24. Bondeven P, Emmertsen KJ, Laurberg S, et al. Neoadjuvant therapy abolishes the functional benefits of a larger rectal remnant, as measured by magnetic resonance imaging after restorative rectal cancer surgery. Eur J Surg Oncol 2015;41:1493-9.

25. Liang Z, Ding W, Chen W, et al. Therapeutic Evaluation of Biofeedback Therapy in the Treatment of Anterior Resection Syndrome After Sphincter-Saving Surgery for Rectal Cancer. Clin Colorectal Cancer 2016;15:e101-7.

26. Rosen HR, Kneist W, Fürst A, et al. Randomized clinical trial of prophylactic transanal irrigation versus supportive therapy to prevent symptoms of low anterior resection syndrome after rectal resection. BJS Open 2019;3:461-5.

27. Ram E, Meyer R, Carter D, et al. The efficacy of sacral neuromodulation in the treatment of low anterior resection syndrome: a systematic review and meta-analysis. Tech Coloproctol 2020;24:803-15.

28. Dulskas A, Kavaliauskas P, Pilipavicius L, et al. Long-term bowel dysfunction following low anterior resection. Sci Rep 2020;10:11882.

29. Sun W, Dou R, Chen J, et al. Impact of Long-Course Neoadjuvant Radiation on Postoperative Low Anterior Resection Syndrome and Quality of Life in Rectal Cancer: Post Hoc Analysis of a Randomized Controlled Trial. Ann
Surg Oncol 2019;26:746-55.

30. Lee SY, Kim CH, Kim YJ, et al. Anastomotic stricture after ultralow anterior resection or intersphincteric resection for very low-lying rectal cancer. Surg Endosc 2018;32:660-6.

31. Polese L, Vecchiato M, Frigo AC, et al. Risk factors for colorectal anastomotic stenoses and their impact on quality of life: what are the lessons to learn? Colorectal Dis 2012;14:e124-8.

32. Celerier B, Denost Q, Van Geluwe B, et al. The risk of definitive stoma formation at 10 years after low and ultralow anterior resection for rectal cancer. Colorectal Dis 2016;18:59-66.

33. Sturiale A, Martellucci J, Zurli L, et al. Long-term functional follow-up after anterior rectal resection for cancer. Int J Colorectal Dis 2017;32:83-8.

34. Emmertsen KJ, Laurberg S. Low anterior resection syndrome score: development and validation of a symptom-based scoring system for bowel dysfunction after low anterior resection for rectal cancer. Ann Surg 2012;255:922-8.

(English Language Editor: A. Kassem)
Cite this article as: Deng $\mathrm{J}, \mathrm{Su} M, \mathrm{Hu} J$, Lin D, Li J, Liu W, Zhang J, Zhong Q, Guo X. A nomogram to predict risk factors of frequent defecation early after ileostomy reversal for rectal cancer patients. Ann Transl Med 2021;9(20):1595. doi: $10.21037 /$ atm-21-4660 